\title{
Calculation of Rail Impedance of Track Circuit Considering Earth Stratification
}

\author{
Bin Zhao ${ }^{{ }^{*}}$, Zhi Jia ${ }^{1}$ and Dong Wang ${ }^{1}$
}

1 School of Automation \& Electrical Engineering, Lanzhou Jiaotong University; zhaobin@mail.lzjtu.cn

\begin{abstract}
Rail impedance directly affects the transmission performance of track circuit. Considering the condition of earth stratification, for the difficult to calculate the rail impedance due to the semiinfinite integration interval and the oscillation of the integrand by using the Carson formula, The truncation method is proposed to divide the impedance formula is divided into definite integral and tail integral. The integral is approximated by the spline function, and the tail integral is calculated by using the exponential integral and Euler formula. Based on it, the rail impedance calculation formula of track circuit is obtained. The electromagnetic field model of track circuit with earth stratification is simulated by finite element method, and the correctness of the method is verified. Based on the formula, the influence of current frequency, soil depth and conductivity on rail impedance is studied. The relative error between the calculated results of rail impedance and the simulation results of finite element is within $5 \%$. It can be seen that the formula has high accuracy and correctly reflects the law of rail impedance variation with current frequency, soil depth and resistivity. It provides a reliable reference for the theoretical calculation of rail impedance of track circuit.
\end{abstract}

Keywords: Rail impedance; earth stratification; Carson formula; Truncation method; Finite element method.

\section{Introduction}

Rail is the carrier of track circuit, which transmits the signal current and traction current, the rail impedance directly affects the transmission performance of track circuit [1]. In the subgrade structure, the rail is laid on the earth, and the electromagnetic characteristics of the soil under different layers are difference. Therefore, it has great significance to accurately calculate the rail impedance of track circuit for transmission performance analysis and optimization design of track circuit [2].

when calculating the rail impedance under the subgrade structure, the rail is aproximated to the overhead line conductor. Carson et al. studied the calculation of overhead conductor impedance with earth as loop, and derived the classical Carson fomula[3,4], which provides a theoretical basis for the calculation of rail impedance. Considering the stratification condition of the earth, Sunde et al. gave the impedance calculation fomula of double-layer large underground, but did not consider the influence of displacement current[5]. Nakagawa et al. [6] proposed the impedance calculation formula for threelayer soil structure considering the influence of relative permittivity, permeability and displacement current of soil. Zou et al. proposed moment function method and asymptotic approximation (ATE) method to solve the problem of unbounded integral interval and integrand oscillation when using Pollaczek formula to calculate overhead conductor impedance[7]. The results show that ate method can improve the convergence speed of Pollaczek integral and reduce calculation time to a certain extent, It is further extended to engineering application. Lee et al. considered the problems of high frequency oscillation and upper limit of semi-infinite integral when calculating the impedance of ground circuit of overhead conductors in horizontal multi-layer underground, divided the impedance expression into two parts, calculated by moment function method and exponential integral method respectively, and obtained the calculation formula of mutual impedance of ground circuit, but ignored the calculation of external impedance, It is not applied to the calculation of rail impedance[8]. 
In order to solve the problem of infinite upper limit integral and high frequency oscillation of integrand function when rail impedance is calculated by Carson formula the truncation method is proposed. At the same time, the electromagnetic field simulation model of track circuit is established, and the rail impedance is calculated by finite element method. By comparing the simulation results with the calculation results in this paper, the correctness of the calculation formula is verified. Based on the formula, the influence of current frequency, soil depth and resistivity on rail impedance is analyzed, which provides a theoretical basis for theoretical calculation of rail impedance in traction power supply system.

\section{Calculation of rail impedance}

Track circuit is an important basic equipment of railway signal, which realizes the automatic control and remote control of modern railway train operation, and uses the rail in the railway line as the carrier to transmit the train control information. By sending and receiving the relevant information of track section, it plays the role of supervising the occupation and clearance of trains and the integrity inspection of track circuit. At the same time, At the same time, it also serves as a channel that can transmit control information, so that the signal, ground equipment and on-board equipment are closely linked. So as to provide an important guarantee for the safe and efficient operation of the train[15].

The schematic diagram of single track line model is shown in Figure 1, which is mainly composed of rail, base and earth. Rail impedance consists of rail self-impedance and mutual impedance. Self-impedance refers to the impedance of the rail itself and ground loop impedance formed by rail and earth, named as internal and external impedance of rail. Mutual impedance refers to impedance formed by electromagnetic induction between two rails and ground loop impedance. In the calculation of rail external impedance, considering the influence of earth eddy current effect, the external impedance should be regarded as two parts. One part refers to the longitudinal impedance of rail (rail and earth are perfect conductors), and the other part refers to the impedance between single rail and earth (earth is lossy medium). In the calculation of internal impedance, because the rail is ferromagnetic material, the theoretical calculation error is large, so the electromagnetic field finite element method is selected for direct simulation. When calculating the mutual impedance, it is necessary to consider the impedance between the two rails and the ground loop formed by each rail with the earth. In the above calculation of rail impedance, the calculation of rail external and mutual impedance is only related to the distribution of rail external magnetic field, but has nothing to do with the distribution of internal electromagnetic field and internal current. The internal impedance of rail is related to rail material (rail is ferromagnetic material) and the distribution of rail internal electromagnetic field. Therefore, considering the calculation of ground loop impedance, the external and mutual impedance of rail are calculated theoretically respectively, and the internal impedance is directly simulated and calculated by finite element method.

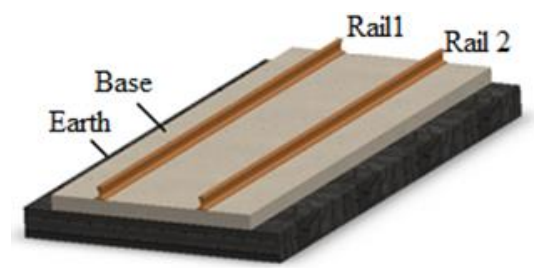

Figure 1. Schematic diagram of single track model

\subsection{Math Calculation of ground loop impedance}

In the actual railway line, the rail is located on the earth, so it can be regarded as overhead line conductor. Therefore, for the calculation of the external and mutual impedance of the rail, the calculation formula for the conductor impedance of the overhead line is used for calculation. At present, Carson ground loop impedance formula is generally 
used to calculate the conductor impedance of overhead lines[16]. It can be seen that the external and mutual impedance of the rail can be expressed as:

$$
\begin{aligned}
& Z_{11}=Z_{11}^{\mathrm{p}}+Z_{11}^{\mathrm{c}} \\
& Z_{12}=Z_{12}^{\mathrm{p}}+Z_{12}^{\mathrm{c}}
\end{aligned}
$$

Where: $Z_{11}$ is the external impedance of the rail, $Z_{12}$ is the mutual impedance of rail, $Z_{11}^{\mathrm{c}}$ and $Z_{12}^{\mathrm{c}}$ are respectively the impedance between a single rail and a ground loop and the mutual impedance between two rails and the earth forming circuit when the earth is the medium of damage, and the calculation formula is:

$$
\begin{gathered}
Z_{11}^{\mathrm{c}}=\mathrm{j} \frac{\omega \mu_{0}}{\pi} \int_{0}^{\infty} F(t) \mathrm{e}^{-2 h_{\mathrm{a}} \lambda} \mathrm{d} t \\
Z_{12}^{\mathrm{c}}=\mathrm{j} \frac{\omega \mu_{0}}{\pi} \int_{0}^{\infty} F(t) \mathrm{e}^{-\left(h_{1}+h_{2}\right) \cdot t} \cos \left(\mathrm{d}_{12} t\right) \mathrm{d} t
\end{gathered}
$$

Where: $t$ is integral variable, $\omega=2 \pi f$ is the angular frequency, $h_{\mathrm{a}}$ is the height of the rail from the ground; $r_{\mathrm{a}}$ is the equivalent radius of rail; $\mu_{0}$ is the vacuum permeability, $\mu_{0}=4 \pi \times 10^{-7} \mathrm{H} / \mathrm{m}, d_{12}$ is the horizontal distance between rail 1 and rail $2, d_{12}=1.435 \mathrm{~m}$.

The impedance of Carson ground loop is closely related to the eddy current effect in the earth, and the inhomogeneity of soil structure will affect the distribution of induced eddy current. Therefore, the calculation of rail external and mutual impedance is related to soil structure. As we all know, eddy current effect will lead to the loss of electromagnetic energy, and the loss will show a trend of attenuation with the increase of surface depth. Under a certain frequency, if the soil structure changes in the range of eddy current loss can't be ignored, the stratification of soil structure in the earth must be considered when the external and mutual impedance of rail is calculated. Based on the two-layer earth structure, this paper calculates the external and mutual impedances of rails. According to equations (1) (4), when the earth is divided into two layers, according to reference [13], the integrand function can be expressed as:

$$
\left\{\begin{array}{l}
F(t)=2 \frac{\left(\mu_{1}+\mu_{2}\right)+\left(\mu_{1}-\mu_{2}\right) \mathrm{e}^{-2 \mu_{1} d}}{\left(\mu_{1}+\mu_{2}\right)\left(\mu_{1}+t\right)+\left(\mu_{1}+\mu_{2}\right)\left(t-\mu_{1}\right) \mathrm{e}^{-2 \mu_{1} d}} \\
\mu_{1}=\sqrt{t^{2}+\frac{\left(\mathrm{j} \omega \mu_{0}\right)^{2}}{\rho_{1}^{2}}} \\
\mu_{2}=\sqrt{t^{2}+\frac{\left(\mathrm{j} \omega \mu_{0}\right)^{2}}{\rho_{2}^{2}}}
\end{array}\right.
$$

Where: $d$ is the depth of the first layer of soil, $\rho_{1}$ is the resistivity of the first layer of soil, $\rho_{2}$ is the resistivity of the second layer of soil.

It can be seen from (3) (5) that it difficult to solve complex integral kernels in the abnormal integral term because of the existence of semi-infinite integral interval and negative exponential factor in the integrand function. Therefore, this paper proposes to use the truncation method to cut the integrand function in the impedance expression into two parts, which are the definite integral and the tail integral with infinite upper limit. At the same time, the moment function and exponential integral function of spline function are used to approximate the truncated definite integral and the tail integral with infinite upper limit.

Firstly, based on the truncation method, (3) and (4) can be simplified as:

$$
\begin{gathered}
Z_{11}^{\mathrm{c} 2}=\mathrm{j} \frac{\omega \mu_{0}}{\pi}\left(\int_{0}^{T} F(t) \mathrm{e}^{-2 h_{\mathrm{a}} \lambda} \mathrm{d} t+\int_{T}^{\infty} F(t) \mathrm{e}^{-2 h_{\mathrm{a}} \lambda} \mathrm{d} t\right) \\
Z_{12}^{\mathrm{c} 2}=\mathrm{j} \frac{\omega \mu_{0}}{\pi}\left(\int_{0}^{T} F(t) \mathrm{e}^{-\left(h_{1}+h_{2}\right) t} \cos \left(\mathrm{d}_{12} t\right) \mathrm{d} t+\int_{T}^{\infty} F(t) \mathrm{e}^{-\left(h_{1}+h_{2}\right) t} \cos \left(\mathrm{d}_{12} t\right) \mathrm{d} t\right)
\end{gathered}
$$

Where: the cut-off value is taken according to reference [16], $T=10 \times \max \left(\left|\gamma_{1}\right|,\left|\gamma_{2}\right|\right)$.

In $[0, \mathrm{~T}], \mathrm{N}+1$ sample points are used to segment the interval, and cubic spline interpolation functio[17] is used to approximate between each two segment points, it can get as: 


$$
\begin{aligned}
& F(t) \approx \sum_{m=1}^{N}\left[\sum_{v=1}^{4} C_{m v}\left(t-t_{m}\right)^{v-1}\right] \Omega\left(t_{m}, t_{m+1}\right) \\
& \Omega\left(\lambda_{m}, \lambda_{m+1}\right)=H\left(\lambda-\lambda_{m+1}\right)-H\left(\lambda-\lambda_{m}\right)
\end{aligned}
$$

Where: $N$ is the number of subintervals; $C_{m v}$ is the fitting coefficient; $\Omega\left(t_{m}, t_{m+1}\right)$ is the window function, $t_{m}$ and $t_{m+1}$ are the end point of the interval; $H\left(\lambda-\lambda_{m+1}\right)$ is the displacement of the Hermitian function[18].

When $t>T$, the Taylor expansion is used to calculate the $\mu_{1}$ and $\mu_{2}$, and $F(t)$ can be simplified to:

$$
F(\lambda) \approx \frac{1}{\lambda}\left[1+\frac{1}{4 \lambda^{2}}\left(\gamma_{1}^{2}-\gamma_{2}^{2}\right) \mathrm{e}^{-2 \mathrm{~d} \lambda}\right]
$$

Let $t=T \xi$, the tail integral be integrated by substitution, and substituting (8) and (10) into (6) and (7) respectively, it can get as:

$$
\begin{aligned}
Z_{12}^{\mathrm{c} 2}= & \mathrm{j} \frac{\omega \mu_{0}}{\pi} \sum_{m=1}^{N} \int_{\lambda_{m}}^{\lambda_{m+1}}\left[\sum_{v=1}^{4} C_{m v}\left(t-t_{m}\right)^{v-1}\right] \mathrm{e}^{-2 h t} \cos \left(\mathrm{d}_{12} t\right) \mathrm{d} t \\
& +\int_{1}^{\infty} \frac{1}{T \xi}\left[1+\frac{1}{4(T \xi)^{2}}\left(\gamma_{1}^{2}-\gamma_{2}^{2}\right) \mathrm{e}^{-2 \mathrm{~d} T \xi}\right] \mathrm{e}^{-2 h T \xi} \cos \left(\mathrm{d}_{12} T \xi\right) \mathrm{d} T \xi \\
Z_{12}^{\mathrm{c} 2}= & \mathrm{j} \frac{\omega \mu_{0}}{\pi} \sum_{m=1}^{N} \int_{\lambda_{m}}^{\lambda_{m+1}}\left[\sum_{v=1}^{4} C_{m v}\left(t-t_{m}\right)^{v-1}\right] \mathrm{e}^{-2 h t} \cos \left(\mathrm{d}_{12} t\right) \mathrm{d} t \\
& +\int_{1}^{\infty} \frac{1}{T \xi}\left[1+\frac{1}{4(T \xi)^{2}}\left(\gamma_{1}^{2}-\gamma_{2}^{2}\right) \mathrm{e}^{-2 \mathrm{~d} T \xi} \mathrm{e}^{-2 h T \xi} \cos \left(\mathrm{d}_{12} T \xi\right) \mathrm{d} T \xi\right.
\end{aligned}
$$

The moment function method can be used to calculate the definite integral in equations (11) and (12).

For tail integral, according to Euler formula [19] and exponential integral function, it can be expressed as:

$$
\begin{gathered}
\mathrm{e}^{-\mathrm{i} T \xi \mathrm{d}_{12}}=\cos \left(T \xi \mathrm{d}_{12}\right)-\mathrm{i} \sin \left(T \mathrm{~d}_{12} \xi\right) \\
\left\{\begin{array}{l}
E_{i}(n, Z)=\int_{1}^{\infty} \exp (-t Z) t^{-n} \mathrm{~d} t \\
n \geq 0, \operatorname{Re}(Z)>0
\end{array}\right.
\end{gathered}
$$

Based on equation (13) and equation (14), the expression of the ground loop impedance in the rail external and mutual impedance of double-layer large underground are obtained as follows:

$$
\begin{aligned}
Z_{11}^{\mathrm{c} 2}= & \mathrm{j} \frac{\omega \mu_{0}}{2 \pi} \sum_{m=1}^{N} \sum_{v=1}^{4} C_{m v} v_{\lambda_{m}}^{\lambda_{m+1}}\left(t-t_{m}\right)^{v-1} \mathrm{e}^{-2 h_{\mathrm{a}} t} \mathrm{~d} t+\mathrm{j} \frac{\omega \mu_{0}}{2 \pi} \operatorname{Re}\left[E_{\mathrm{i}}\left(1,2 h_{\mathrm{a}} T\right)\right] \\
& +\mathrm{j} \frac{\omega \mu_{0}}{2 \pi} \frac{\gamma_{1}^{2}-\gamma_{2}^{2}}{4 T^{2}} \operatorname{Re}\left[E_{\mathrm{i}}\left(3,2\left(\mathrm{~d}_{12}+h_{\mathrm{a}}\right) T\right)\right] \\
Z_{12}^{\mathrm{c} 2}= & \mathrm{j} \frac{\omega \mu_{0}}{2 \pi} \sum_{m=1}^{N} \sum_{v=1}^{4} C_{c v} \operatorname{Re}\left[\int_{\lambda_{m}}^{\lambda_{m+1}}\left(\lambda-\lambda_{m}\right)^{v-1} \mathrm{e}^{-\delta \lambda} \mathrm{d} \lambda\right] \\
& +\mathrm{j} \frac{\omega \mu_{0}}{2 \pi}\left\{\operatorname{Re}\left[E_{\mathrm{i}}(1, \delta T)\right]+\frac{\gamma_{1}^{2}-\gamma_{2}^{2}}{4 T^{2}} \operatorname{Re}\left[E_{\mathrm{i}}(3, \beta T)\right]\right\}
\end{aligned}
$$

Where: $\beta=2 d+2 h+\mathrm{i} x, \delta=2 h+\mathrm{i} x$.

\subsection{Calculation of rail external impedance}

Taking a single rail as the research object, the external impedance of the rail is calculated. The calculation model of the external impedance is shown in Figure 2. 


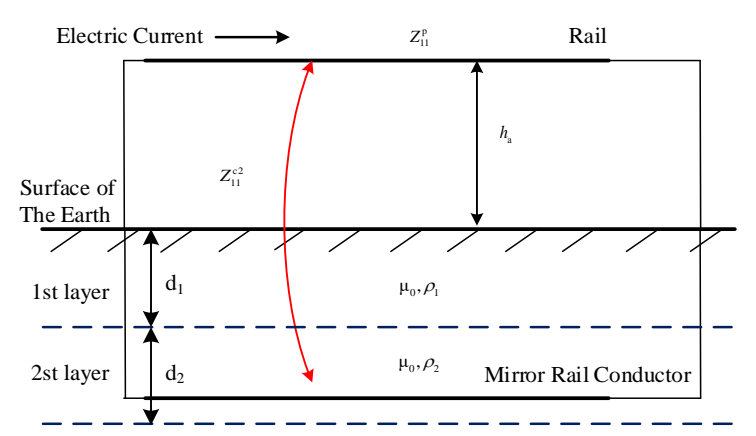

Figure 2. Calculation model of rail external impedance

According to Carson formula, the longitudinal impedance $Z_{11}^{\mathrm{p}}$ is:

$$
Z_{11}^{\mathrm{p}}=\mathrm{j} \frac{\omega \mu_{0}}{2 \pi} \ln \frac{2 h_{\mathrm{a}}}{r_{\mathrm{a}}}(17)
$$

According to (1) and sorting out (15) and (17), the calculation formula of rail external impedance under the condition of double-layer earth $Z_{11}^{2}$ is obtained as follows:

$$
\begin{aligned}
Z_{11}^{2}= & \frac{\omega \mu_{0}}{2 \pi} \ln \frac{2 h_{\mathrm{a}}}{r_{\mathrm{a}}}+\mathrm{j} \frac{\omega \mu_{0}}{2 \pi} \sum_{m=1}^{N} \sum_{v=1}^{4} C_{m v} \int_{\lambda_{m}}^{\lambda_{m+1}}\left(t-t_{m}\right)^{v-1} \mathrm{e}^{-2 h_{\mathrm{a}} t} \mathrm{~d} t+\mathrm{j} \omega \frac{\mu_{0}}{2 \pi} \operatorname{Re}\left[E_{\mathrm{i}}\left(1,2 h_{\mathrm{a}} T\right)\right] \\
& +\mathrm{j} \frac{\omega \mu_{0}}{2 \pi} \frac{\gamma_{1}^{2}-\gamma_{2}^{2}}{4 T^{2}} \operatorname{Re}\left[E_{\mathrm{i}}\left(3,2\left(\mathrm{~d}_{12}+h_{\mathrm{a}}\right) T\right)\right]
\end{aligned}
$$

\subsection{Calculation of rail mutual impedance}

The calculation of rail mutual impedance consists of two parts. One part is generated by the mutual influence of the magnetic fields of two rails, which is expressed by $Z_{12}^{\mathrm{p}}$ and calculated by Carson formula; The other part is the interaction between the loop formed by the rail and the earth, which is expressed by $Z_{12}^{\mathrm{c}}$ and calculated by Carson's impedance formula of the earth loop. The calculation model of rail mutual impedance is shown in Figure 3. $Z_{12}^{\mathrm{p}}$ is the mutual impedance between rails when rail and earth are perfect conductors, The calculation formula is as follows:

$$
\left\{\begin{array}{l}
Z_{12}^{\mathrm{p}}=\mathrm{j} \frac{\omega \mu_{0}}{2 \pi} \ln \frac{D_{12}}{\mathrm{~d}_{12}} \\
D_{12}=\sqrt{\mathrm{d}_{12}^{2}+\left(h_{1}+h_{2}\right)^{2}}
\end{array}\right.
$$

Where: $h_{1}$ and $h_{2}$ are the height of each rail above ground respectively; $D_{12}$ is the distance between the mirror conductor of rail 1 and rail 2 .

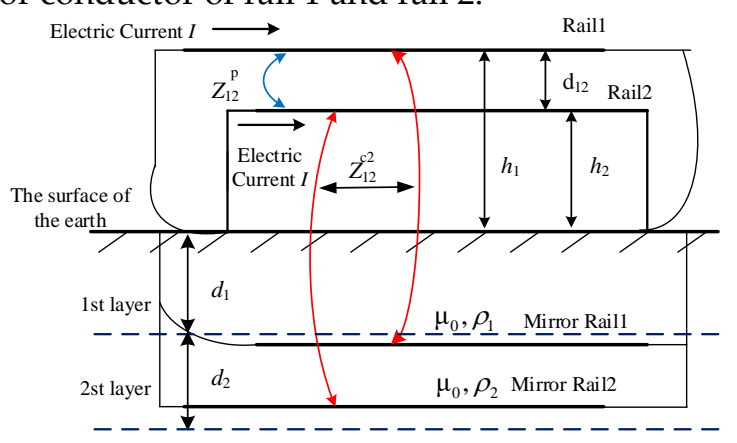

Figure 3. Calculation model of rail mutual impedance

According to (2) , (16) and (19) , the calculation formula of rail mutual impedance $Z_{12}^{2}$ in the case of double-layer earth is obtained as follows: 


$$
\begin{aligned}
Z_{12}^{2}= & Z_{12}^{\mathrm{p}}+Z_{12}^{\mathrm{c} 2} \\
= & \mathrm{j} \frac{\omega \mu_{0}}{2 \pi} \ln \frac{D_{12}}{d_{12}}+\mathrm{j} \frac{\omega \mu_{0}}{2 \pi} \sum_{m=1}^{N} \sum_{v=1}^{4} C_{c v} \operatorname{Re}\left[\int_{\lambda_{m}}^{\lambda_{m+1}}\left(\lambda-\lambda_{m}\right)^{v-1} \mathrm{e}^{-\delta \lambda} \mathrm{d} \lambda\right] \\
& +\mathrm{j} \frac{\omega \mu_{0}}{2 \pi}\left\{\operatorname{Re}\left[E_{\mathrm{i}}(1, \delta T)\right]+\frac{\gamma_{1}^{2}-\gamma_{2}^{2}}{4 T^{2}} \operatorname{Re}\left[E_{\mathrm{i}}(3, \beta T)\right]\right\}
\end{aligned}
$$

Since the function can be calculated analytically, and the exponential function can also be calculated by MATLAB, the calculation is simple and convenient. Based on this method, the problem of high frequency oscillation of integral kernel function and difficulty in solving can be avoided when Carson impedance formula is used to calculate rail impedance. It can also be used to calculate rail external impedance and mutual impedance under double-layer earth condition.

\section{Finite element simulation}

The self-impedance and mutual impedance of BS113A rail are simulated by the finite element method, the results show that it has high accuracy to calculate the rail impedance[9]. The finite element simulation results as a reference to verify the correctness of rail internal impedance [20]. According to the electromagnetic field simulation model of track circuit and the structure of ballasted track, the rail impedance is simulated by multi physical field coupling software COMSOL. Because the track line is located in infinite open area, there are some errors in the direct simulation calculation. In order to improve the accuracy of the calculation results, it is necessary to deal with the infinite open area. In the simulation, the infinite open area is transformed into a finite area by using the cylindrical coordinate transformation of the infinite element. Because the rail is a nonlinear ferromagnetic material, it is easy to be magnetized and has hysteresis. For the accuracy of the calculation results, considering the nonlinear change of permeability, the material properties of P60 rail B-H magnetization curve in reference [21] and rail, earth and other material parameters in reference [12] are set. The conductivity of rail is $6 \times 10^{6} \mathrm{~s} / \mathrm{m}$, the first layer is $0.1 \mathrm{~s} / \mathrm{m}$ and the second layer is $0.01 \mathrm{~s} / \mathrm{m}$. Considering the skin effect of the rail, the physical field is added as the magnetic field, and the frequency domain research is carried out. The excitation is set to $50 \mathrm{~Hz}$ traction return current. The influence of skin depth determined by conductivity and current frequency on model mesh generation, rail mesh generation and skin effect results are shown in Fiure4(a) and Fiure4(b). The mesh generation of P60 rail model is shown in Figure 5 and Figure 6. The self-impedance and mutual impedance of rail can be obtained by volume integration of ohmic loss and magnetic energy storage.

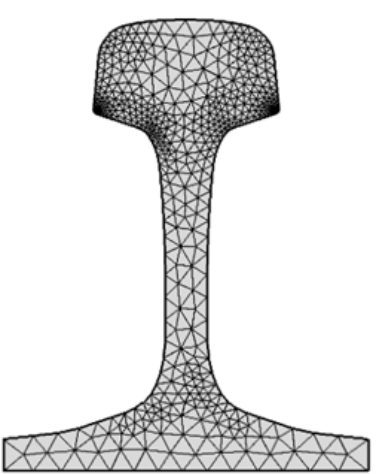

(a) Grid generation of rail section

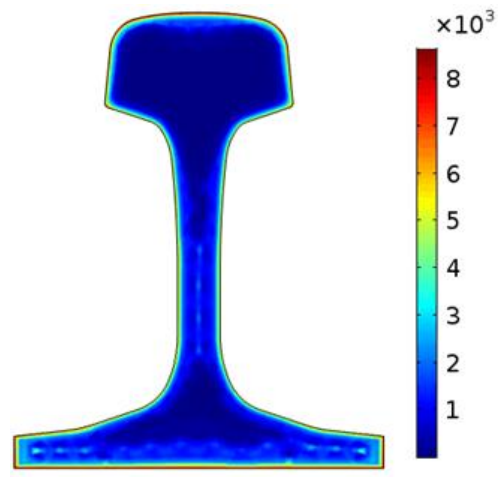

(b) Skin effect of rail

Figure 4. Mesh generation and skin effect of rail section 


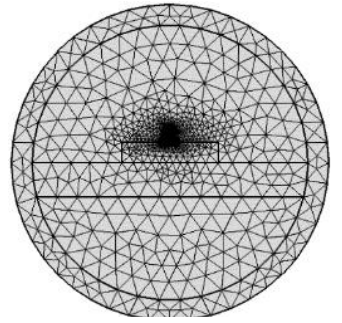

Figure 5. Mesh generation of external impedance

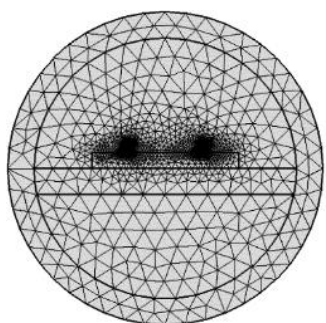

Figure 6. Mesh generation of mutual impedance

Considering the layered structure of the earth, based on the finite element method, the external and mutual impedance electromagnetic loss diagrams are shown in Figure7 and Figure8. It can be seen from Figure 7 and Figure 8 that the electromagnetic loss of rail and earth is the most, followed by that of concrete base. In the earth loss, the loss of the first layer of earth structure is greater than that of the second layer, while the loss of air and infinite element domain is the least. This is because the eddy current loss is mainly related to the conductivity of the conductor, and the greater conductivity of the conductor, the greater energy loss. However, the conductivity values of rail, earth, track plate and air are decreasing, and the conductivity of the first layer of earth is greater than that of the second layer, which leads to the electromagnetic energy loss to appear unevenly distributed.

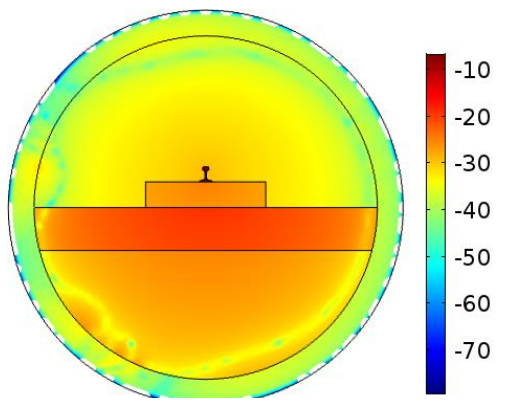

Figure 7. Electromagnetic loss diagram of

layered external impedance model

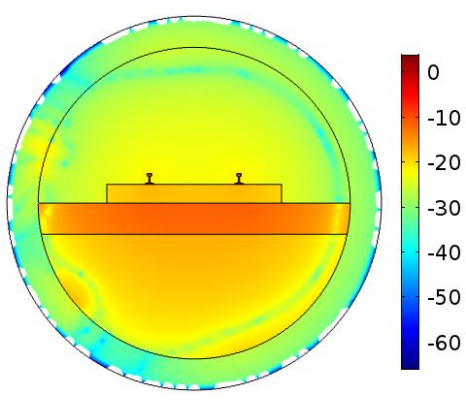

Figure8. Electromagnetic loss diagram of layered earth mutual impedance model

Because of the I-shape of rail section, the rail external impedance can't be calclated directly, it can be calculated as a cylindrical conductor by using the principle of perimter equivalence. According to the relevant parameters of P60 rail [22], the equivalent radius is $0.1066 \mathrm{~m}$, and the thickness of the first soil layer is $20 \mathrm{~m}$. In the electrified section, not only the traction current but also the signal current flows through the rail. The frequency of signal current of track circuit is $1700 \sim 2600 \mathrm{~Hz}$, while the frequency of traction current is $50 \mathrm{~Hz}$. Therefore, the current frequency is $0 \mathrm{~Hz} 10000 \mathrm{~Hz}$.

Due to the complex environment of track line and the limitation of experimental conditions, it is difficult to measure directly. Therefore, this paper uses the finite element method to verify the accuracy of the theoretical formula of rail impedance. Based on the above parameters, the rail impedance is calculated by the proposed method and the finite element method. Because the internal impedance calculation in this paper is directly based on the finite element method, it is only necessary to verify the theoretical calculation formula of rail external and mutual impedance. Based on the results of finite element simulation, the relative error is calculated. The formula of relative error is as follows:

$$
\delta=\frac{\left|y-y_{0}\right|}{y} \times 100 \%
$$

The rail external and mutual impedances calculated by this algorithm are expressed in $y$; The external and mutual impedances of rails calculated by the finite element method are expressed in $y_{0}$. Based on equation (18) and equation (20) and finite element method, the external impedance and mutual impedance of rail under double-layer earth are calculated. The calculation results are shown in Figure9. 


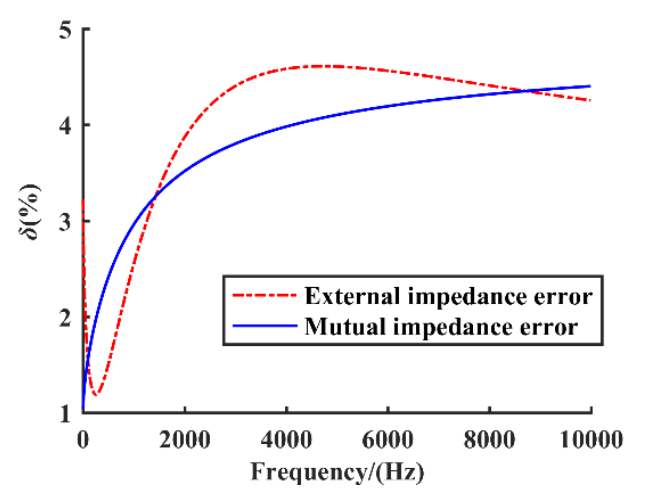

Figure9. Error of double-layer external impedance and mutual impedance

It can be seen from figure 9 that the calculation results of this method and the finite element simulation results have a certain error in the current frequency range of $0 \mathrm{~Hz} \sim 10 \mathrm{kHz}$, and the maximum error is within $5 \%$. This is because in the part of theoretical calculation, this paper uses the approximate idea of moment function method to calculate Carson's earth circuit impedance formula, that is, expanding the integrand function at each point into a cubic coefficient polynomial for approximate calculation, which causes certain errors. Moreover, when the finite element method is used, its solution domain setting and infinite element domain equivalence will have a certain impact on the simulation results. Therefore, the calculation method in this paper can accurately calculate the external and mutual impedance of double-layer large underground rail.

\section{Analysis of influencing factors}

\subsection{Effect of current frequency}

There are mainly two kinds of current in the rail line, which make the current frequency present a wide frequency distribution. For example, the signal current is usually several thousand Hertz, while the traction current is generally $50 \mathrm{~Hz}$. Therefore, this method is used to study the variation of rail self impedance and mutual impedance with current frequency from $0 \mathrm{~Hz}$ to $10000 \mathrm{~Hz}$. In the calculation of rail self impedance and mutual impedance, the calculation results include real part and imaginary part. In the impedance formula, the real part represents resistance and the imaginary part represents inductance. Therefore, the real part of self impedance and mutual impedance is defined as self resistance and mutual resistance respectively, and the imaginary part of self impedance and mutual impedance is defined as self inductance and mutual inductance respectively. The variation curves of rail self and mutual impedance with frequency are shown in figure10 and figure11.

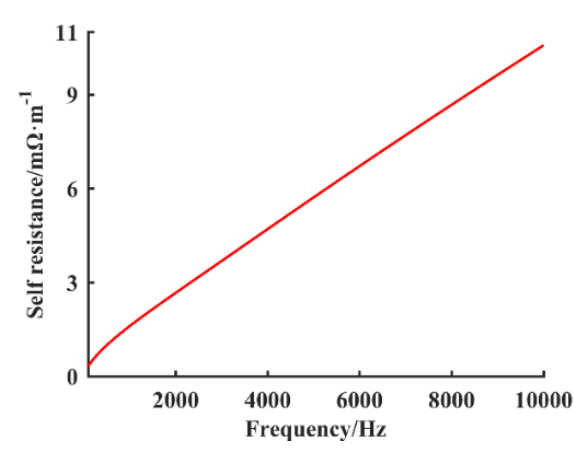

(a)Variation of self resistance with frequency

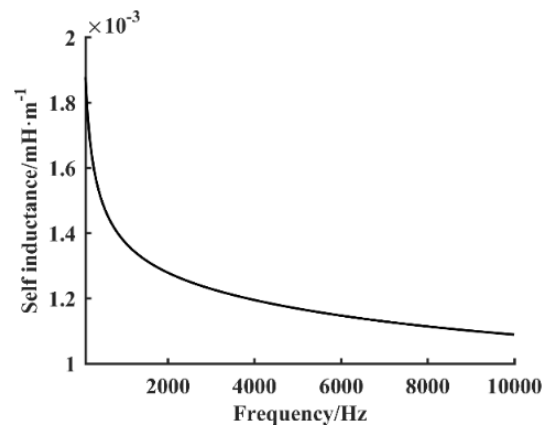

(b) Variation of self inductance with frequency

Figure10. Variation of rail self impedance with frequency 


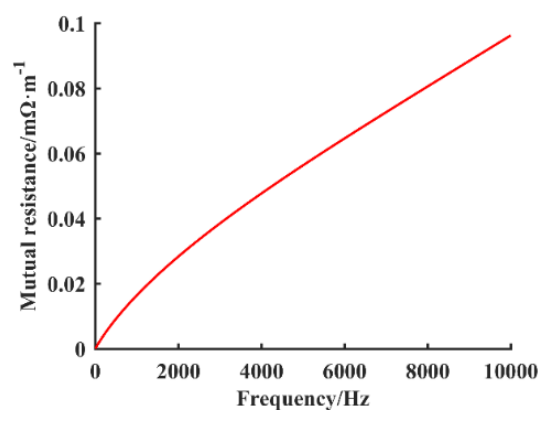

(a)Variation of mutual resistance with frequency

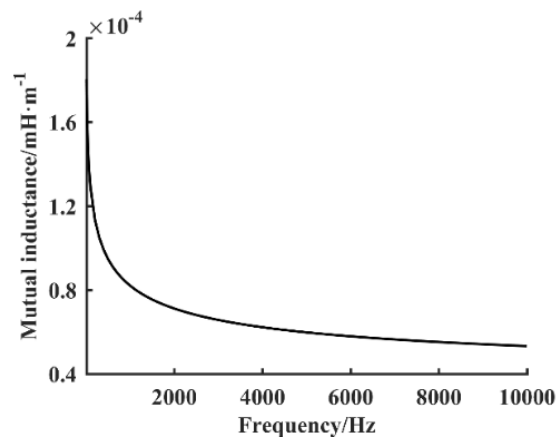

(b) Variation of mutual inductance with frequency

Figure11. Variation of rail mutual impedance with frequency

It can be seen from Figure. 10 and Figure. 11 that the rail self impedance and mutual impedance change nonlinearly with frequency. Specifically, the rail self resistance and mutual resistance change positively with frequency, slowly at low frequency, and significantly at high frequency. The rail mutual inductance changes negatively with the increase of frequency, and the curve changes obviously at low frequency. With the increase of frequency, the change rate gradually decreases and tends to be stable. This is because the eddy current effect of the earth plays a demagnetizing role, and the larger the frequency is, the stronger the demagnetizing effect is, and the smaller the mutual inductance is.

\subsection{Effect of soil depth}

China's vast territory and complex geological distribution lead to the diversity of rail laying environment (bridge, tunnel, subgrade). The different structure of track and earth will affect the calculation of rail impedance of track circuit, which will change the transmission performance of track circuit. For the subgrade section, the height of the ground where the rail is laid and the soil environment in the area where the train passes will be different. Therefore, not only the influence of current frequency on rail impedance, but also the change of soil depth and soil conductivity should be considered. Doublelayer earth is taken as an example, the first layer is finite depth, and the second layer is infinite depth. The variation of rail self impedance and mutual impedance of ballastless track circuit with the depth of the first layer of soil is given and analyzed.

The self impedance and mutual impedance of ballastless track circuit rail are calculated when the current frequency is $1700 \mathrm{~Hz}, 2000 \mathrm{~Hz}, 2300 \mathrm{~Hz}$ and $2600 \mathrm{~Hz}$ respectively by taking the first layer of soil depth from $5 \mathrm{~m}$ to $50 \mathrm{~m}$. Figure. 12 and Figure. 13 show the variation curves of rail self impedance and mutual impedance with the depth of the first layer of soil respectively.

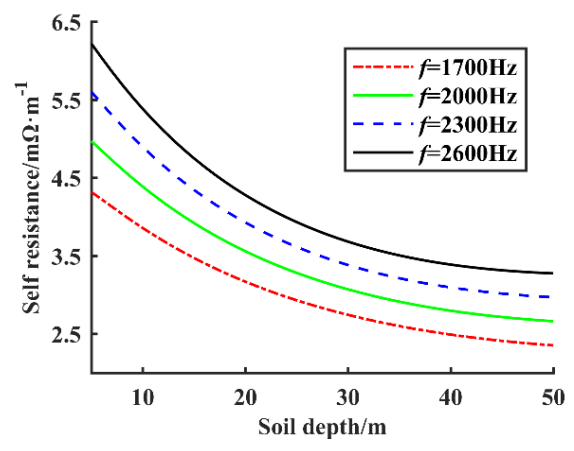

(a) Self resistance changes with soil depth

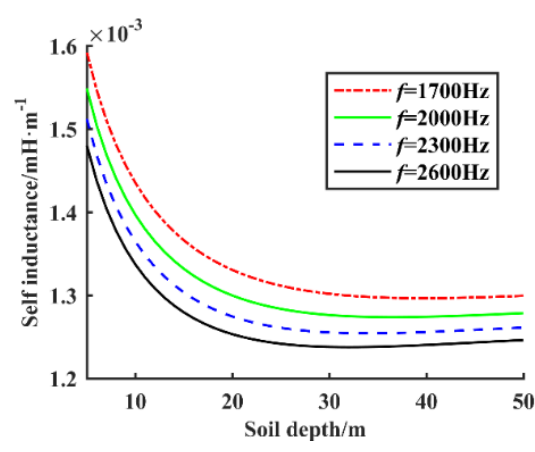

(b) Self inductance changes with soil depth

Figure. 12 Variation of rail self impedance with soil depth 
It can be seen from Figure12 and Figure13 that the rail self impedance and mutual impedance decrease with the increase of the first layer of soil depth. When the soil depth of the first layer is small, the external resistance and inductance of the rail decrease rapidly and change obviously. With the increase of soil depth, the change rates of external resistance and inductance tend to be flat. This is because the electromagnetic wave will change rapidly with the increase of the depth of the first layer of soil, and then gradually attenuate with the increase of the depth, and finally tend to saturation. At the same soil depth, the rail self impedance and mutual impedance vary with frequency. Among them, the rail self resistance and mutual resistance are positively correlated with the increase of frequency, while the rail self inductance and mutual inductance are negatively correlated with the increase of frequency. It can be seen that the first soil depth has a greater impact on the rail self impedance and a smaller impact on the rail mutual impedance.

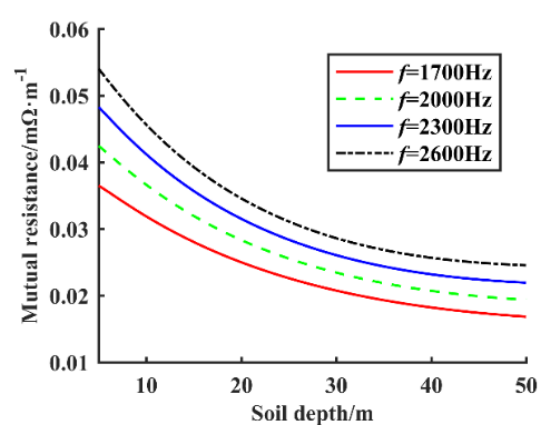

(a)Mutual resistance changes with soil depth

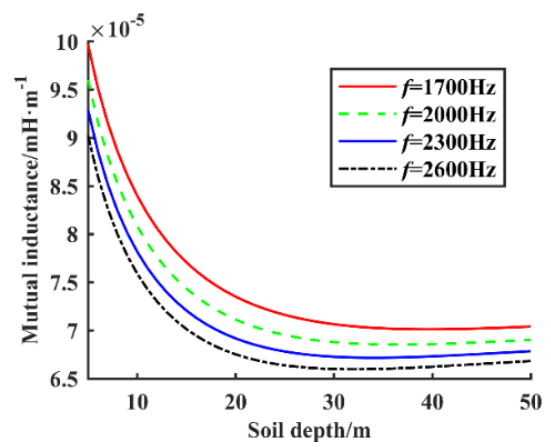

(b)Mutual inductance changes with soil depth

Figure 13.Variation of rail mutual impedance with soil depth

\subsection{Effect of soil resistance}

Taking the first layer of earth resistivity Q1 and the second layer of earth resistivity Q2 in the range of $0 \Omega / \mathrm{m} \sim 100 \Omega / \mathrm{m}$. The variation of rail self impedance and mutual impedance with the resistivity of two layers of soil is shown in Figure14 and Figure15.

From figure.14 and figure.15, it can be seen that the self impedance and mutual impedance of rail are nonlinear with the soil resistivity of the first and second layers. When the second layer of soil resistivity is fixed, the self impedance and mutual impedance of rail show positive correlation with the increase of the first layer soil resistivity, and the increasing trend of the first layer soil resistivity gradually slows down. When the resistivity of the first layer of soil is a certain value, the self impedance and mutual impedance of rail increase with the increase of the resistivity of the subsoil, but the change is slow. It can be concluded that the self impedance and mutual impedance of rail are greatly affected by the soil resistivity of the first layer.

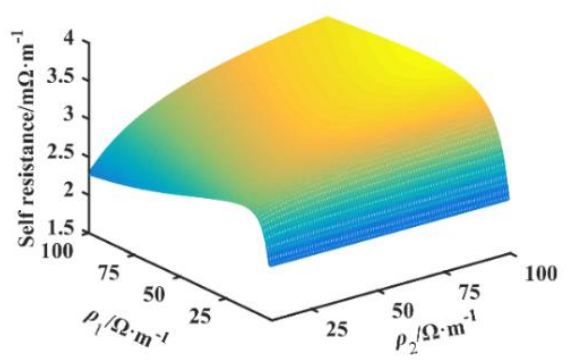

(a)Variation of self resistance with earth resistivity

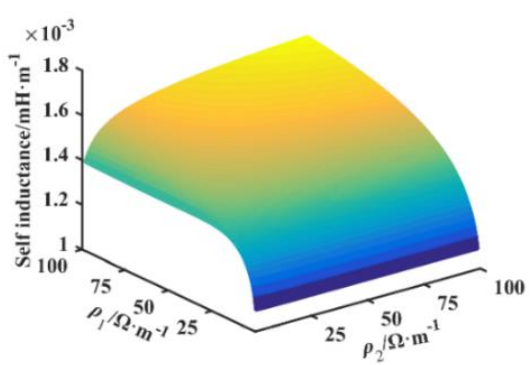

(b) Vriation of self inductan with earth resistivity

Figure 14. variation of rail self impedance with earth resistivity 


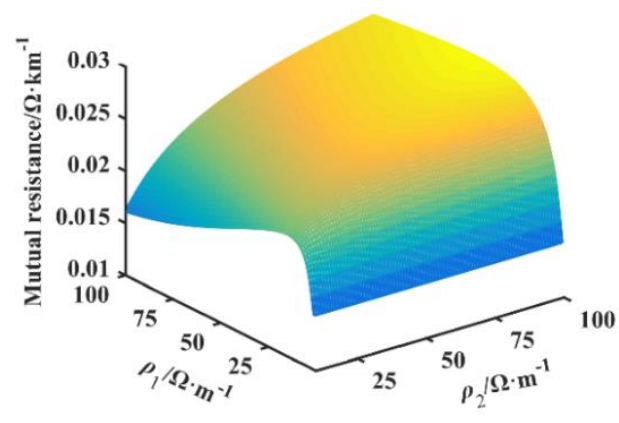

(a) Variation of mutual resistance with earth resistivity

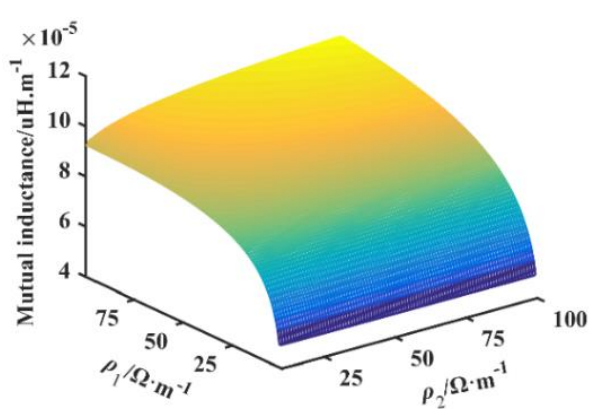

(b) Variation of mutual inductance with earth resistivity

Figure 15. Variation of rail mutual impedance with earth resistivity

\section{Conclusions}

(1) In order to solve the problems of infinite upper limit integral and high frequency oscillation of integrand when using Carson formula, the formula is divided into two parts: definite integral and tail integral by truncation method, and the moment function method and exponential integral method are used to calculate respectively, and the theoretical calculation formula of layered large underground rail impedance is obtained.

(2) The results show that the rail self-resistance and mutual resistance increase with the increase of frequency, while the rail self-inductance and mutual inductance decrease with the increase of frequency; The self-impedance and mutual impedance of rail decrease with the increase of the first layer depth; The first layer of soil resistivity has great influence on rail self-impedance and mutual impedance.

(3) This method can accurately calculate the rail impedance and provide theoretical calculation formula for the analysis of transmission performance of track circuit and the modeling of traction network system.

Funding: This research was funded by the National Natural Science Foundation of China under Grant 51967010.

\section{References}

1. Chang, Li Nan, Zhang Yadong, et al. Combination test sequence reduction of railway signal safety critical software, Journal of Southwest Jiaotong University,2020,vol.253,no.3,pp. 149-156.

2. Zhang Youpeng, Wang Dong, Zhao Bin, et al. Study on rail self impedance calculation of track circuit considering earth influence, Journal of Railway Science and engineering,2020, vol.128,no.11,pp. 225-233.

3. John R. Carson. Wave propagation in overhead wires with ground return, Bell Labs Technical Journal, 1926,vol.5,no.4,pp. 539-554.

4. Zhang Youpeng, Wang Dong, Zhao Bin, et al. Study on rail self impedance calculation of track circuit considering earth influence , Journal of Railway Science and engineering,2020, vol.128,no.11,pp. 225-233.

5. John R. Carson. Wave propagation in overhead wires with ground return, Bell Labs Technical Journal, 1926,vol.5,no.4,pp. 539-554.

6. Papagiannis G.K, Tsiamitros D.A, Labridis D.P, et al . A systematic approach to the evaluation of the influence of multilayered earth on overhead power transmission lines, IEEE Transactions on Power Delivery, 2005,vol.20,no.4,pp. 2594-2601.

7. Butler-Purry, Karen. Distribution system modeling and analysis. IEEE Power \& Energy Magazine, 2020,vol.11,no.3,pp. 106-108.

8. Deri A, Tevan G, Semlyen A, et al . The complex ground return plane a simplified model for homogeneous and multi-Layer earth return, IEEE Power Engineering Review, 1981,vol.1,no.8,pp. 31-32.

9. Hill R. J, Carpenter D. C. Determination of rail internal impedance for electric railway traction system simulation,IEE Proceedings. Part B, Electric Power Applications, vol.138,no.6,pp. 311-321.

10. Hill R J, Brillante S, Leonard P J . Railway Track Transmission Line Parameters From Finite Element Field Modelling: Series Impedance,IEE Proceedings-Electric Power Applications, 2000, vol.146, no.3,pp. 227-238.

11. Hill R J, Carpenter D C. Modelling of nonlinear rail impedance in AC traction power systems,IEEE Transactions on Power Delivery, 1991 ,vol.6,no.4,pp. 1755-1761.

12. Hill R J, Carpenter D C. Rail track distributed transmission line impedance and admittance: theoretical modeling and experimental results. IEEE Transactions on Vehicular Technology, 1993,vol.42,no.2,pp. 225-241.

13. Sunde E D. Earth Conduction Effects in Transmission Systems, Students Quarterly Journal, 1949,vol.20,no.78,pp. 92. 
14. Nakagawa M, Ametani A, Iwamoto K. Further studies on wave propagation in overhead lines with earth return: impedance of stratified earth. Proceedings of the Institution of Electrical Engineers, 1973,vol.120,no.12,pp. 1521-1528.

15. ZHU Jun, WU Guangning, GONG Hongkui, et al . Calculation and study of impedance of conductor-earth circuits for overhead line systems with layered soil structure,High Voltage Apparatus, 2014,vol.50,no.2,pp. 29-34.

16. Lee J B ,Zou J , Li B ,et al. Efficient evaluation of the earth return mutual impedance of overhead conductors over a horizontally multilayered soil. Compel International Journal for Computation \& Mathematics in Electrical \& Electronic Engineering, 2014,vol.33,no.4,pp. 1379-1395.

17. Wang Shisong. Measures to prevent short circuit accident of track circuit, Railway architecture, no.8,pp. 21-24 (1981).

18. Krolo I, Modric T, Vujevic S. Definition and Computation of Carson Formulas, InternationalMultidisciplinary Conference on Computer \& Energy Science,Split, Croatia Republic, 2017 ,pp. 1 .

19. Gao Xiaoke, Deng Zichen, Huang Yong'an. Precise integration method based on cubic spline interpolation,Vibration and shock, 2007,vol.26,no.9,pp. 75.

20. Wang Xinyu. Image super resolution method based on sparse regularization and repeated purification, PhD Thesis, University of Electronic Science and technology, 2016,Chengdu,China.

21. Yin Jiantang. Euler formula and its application, Mathematical communication, 2006 ,no.8,pp. 4.

22. ZHU Feng,LI Jiacheng,LI Pengzhen, etal . Calculation of Alternating-current Impedance for the Rail of Electric Railway, Journal of the China Railway Society, 2017 ,vol.39,no.12,pp. 38-42.

23. Liu siran, Chen Jianyun, LEGO. Calculation of impedance frequency dependent parameter matrix of traction network considering rail ferromagnetic characteristics, Journal of East China Jiaotong University, 2018, vol.35,no.4,pp. 97-105.

24. Zhu Feng, Li Xin, Li Pengzhen, etal. Accurate calculation formula of rail inductive reactance of electrified railway, China Railway Science, 2017,vol.38,no.3,pp. 83-88 . 